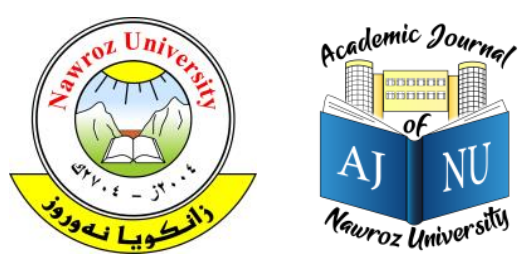

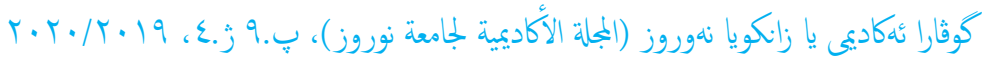

حقوق الطبع والنشر (2017. هذه مقالة الوصول اليها مفتوح موزعة تحت رخصة

e-ISSN: 2520-789X ،CC BY-NC-ND 4. 0. - المشاع الايداعي النسبي

https://doi.org/10.25007/ajnu.v9n4a917

\title{
أثر بعض مثغيرات الاقتصاد الكلي في المؤشر العام لسوق العراق للأوراق المالية \\ للمدة (2005-2018): دراسة تطبيقية
}

ح.م. هلزين مردان مصطفى، قسم الاقتصاد، كلية الادارة والاقتصاد، جامعة دهوك، إقليم كوردستان العراق

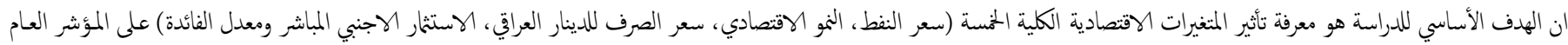

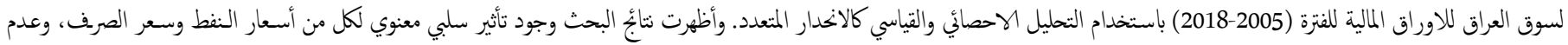

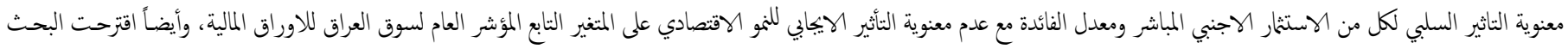

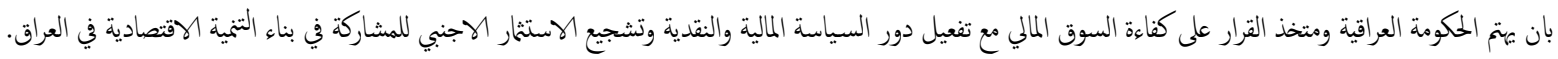
الكلمات الدالة: السوق المالي، أسعار النفط، النمو الاقتصادي، الاستثار الاجنبي المباشر، سعر الصرف.

واستقرارها من خلال ممارسة وظائفها بشكل صيح كوسيط مالي لتوجيه الوحدات المدخرة للاستثمار باتجاه وحدات العجز سيساهم في الازدهار الاقتصادي. يعتبر نظرية التسعير بالمرابحة (Arbitrage Pricing Theory) التي وضعها هي القاعدة الاساسية التي بنيت عليها هذا البحث، والتي تضمنت (Ross, 1976) بان النموذج متوازن لكونها تسمح بأن يتم تحقيق التوازن في العائد على الموجودات بالاعتماد على عوامل عديدة وليس عامل واحد فقط، حيث تفتزض هذه النظرية بأن العائد على الموجودات هي دالة خطية من عوامل الاقتصاد الكلي المختلفة أو نظرياً مؤشرات السوق المالي، وتنص النظرية على أن العائد المحقق على الموجودات يتكون من العائد المتوقع على ذلك الموجود في بداية الفترة الزمنية والادراك الغير متوقع لعوامل الخطر خلال تلك الفترة الزمنية بالإضافة إلى المخاطر الخاصة بالشركة. ولذلك فأن هدف البحث هو تحليل آثار بعض متغيرات الاقتصاد الكلي على المؤشر العام لسوق العراق للاوراق المالية في إطار نظرية التسعير بالمرابحة، حيث تح تحليل مؤشر السوق بالاعتماد على البيانات السنوية للمدة (2005-2018)، ومتغيرات الاقتصاد الكلي المستخدمة المخسة هي سعر النفط، النمو الاقتصادي، سعر الصرف، نسبة الاستثمر الاجنبي المباشر الم اجالي الناتج المحلي ومعدل الفائدة. تم استخدام طريقة الانحدار الخطي المتعدد في تحليل السلاسل الزمنية. ما تبقى من

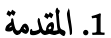

يشير السوق المالي الى مجموعة من التسهيلات والترتبات والاجراءات المؤسسية لتوفير الاقراض والاقتزاض من قبل الوحدات الاقتصادية (غير المالية) وتقوم بدور الوسطاء الماليين من أجل تسهيل عملية تويل على المدى المتوسط وطويل الاجل، faure, ) بمعنى أخر يمارس دور الوسيط بين وحدات العجز ووحدات الفائض 8 ,2013)، وبالتالي يعرف سوق رأس المال على أنه آلية منظمة ذو كفاءة وفاعلية في تحويل رأس المال أو الموارد المالية من الأفراد أو مؤسسات الادخار إلى القطاعات الاقتصادية الخختلفة ومنها الصناعي، وإن سوق رأس المال الفعال يعتمد على توفير المدخرات، والنظام المالي الجيد ومجموعة من الاشخاص أصحاب الأعمال الريادية، باعتبار سوق رأس المال هو سوق لتوفير الأموال على المدى الطويل، بينما تقوم سوق النقد بتوفير الأموال في المدى القصير.

إن السوق المالي تساهم بشكل كبير في عملية التنمية والنمو الاقتصادي من خلال تشجيع الاستثمار وتحفيز المدخرات على مستوى الافراد والشركات والحكومات، ولا سيا أن الدور الذي تمارسه الاسواق المالية ضروري جدا لتحقيق الاستقرار في القطاع الملالي وتوفير قناة استثمرية محمة تساهم في جذب رؤوس الأموال المحلية والأجنبية وخاصة في الدول النامية ومنها العراق باعتبارها ذو اقتصاد ريعي معتمد على النفط فقط، وبالتالي فان تطوير وصعود اداء سوق العراق للاوراق المالية 


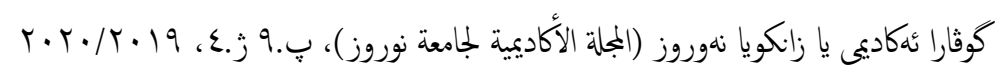

إن معرفة العلاقة التطبيقة بين متغيرات الاقتصاد الكلي والسوق المالي في العراق غير واضحة المعالم لحد الآن نتيجة لعدم وجود دراسات عديدة حول الموضوع، وقد يعود ذلك الى حداثة السوق الملالي في العراق وخاصة من ناحية استقلالها التي بدات بعد العام (2004)، واستقبل العراق تدفقات الاستثمارات الاجنبية. وعلى الرغز من وجود العديد من الدراسات السابقة في الدول المتقدمة والنامية التي ربطت بين متغيرات الاقتصاد الكلي والاسواق المالية الا أن النتائُ كانت متفاوتة وغيرحسومة لحد الان (المعموري والزبيدي، 2014: حسن وصباح، 2019: باش والقريشي، 2017)، بالاضافة الى كون العراق يتسم بعدم الاستقرار الامني والسياسي وارتفاع النشاطات الارهابية وخاصة في السنوات الاخيرة بعد ظهور داعش، والتي أدت الى عدم الاستقرار في متغيرات الاقتصاد الكلي وانغكسها على النمو الاقتصادي والسوق المالي (Asaad, 2014,193). لذلك فإن هذا البحث جاءت لتغطية الفجوة الموجودة في الدراسات السابقة عن السوق المالي في العراق (Asaad, 2015, 77)، بالاضافة الى عدم جذب الاستثخرات الاجنبية المباشرة يرجع الى تدمير البنية التحتية والفساد الاداري والمالي وغياب البيئة القانونية وفشل السياسة المالية والنقدية (خلف، 2013)، وأن قيمة الأسهم تتأثر بشكل كير بالمتغرات الاقتصادية نتيجة تفاعل السوق المالي مع البيئة الاقتصادية كالعرض والطلب على الأسهم ومعدل التضخم وأسعار الفائدة والنمو الاقتصادي (المرشدي، 2018، 189) (المعموري والزبيدي، 2014: حسن وصباح، 2019: باش والقريشي، 2017)، لذلك يفترض الاجابة على التساؤل الاتي: هل هناك تأثير لبعض متغيرات الاقتصاد الكلي على

$$
\begin{array}{r}
3.1 \\
\text { أهداف البعاق للاوراق المالية؟. }
\end{array}
$$

- - دراسة اثر متغيرات الاقتصاد الكلي (سعر النفط، النمو الاقتصادي، سعر الصرف، نسة الاستثار الاجنبي المباشر الى اجلالي الناتج المحلي ومعدل الفائدة) على المؤشر العام لسوق العراق للأوراق المالية للفترة (2005-

.(2018
الدارسة نظمت في ثلاثة مباحث فالاول يتضمن الجانب النظري للاقتصاد الكلي والسوق المالي وبعض الدراسات السابقة، والمبحث الثاني تركز على مناقثة نتاجُ التحليل لتأثير بعض متغيرات الاقتصاد الكلي على السوق المالي في العراق للفترة (2018-2005)، بينا المبحث الثالث والاخير قدم بموعة من الاستنتاجات

$$
\begin{array}{r}
1.1 \\
\text { والمقترحات البحث أهمية البحث من عدة جوانب ومنها: }
\end{array}
$$

- اعتبار السوق الملالي أخذت هيزاً كبيرا وكامل مم لجذب الاستثمار والادخار وتحقيق المنافسة في السوق، وبذلك تأتي أهمية البحث من ناحية الاهنمام القليل بتاثير متغيرات الاقتصادي الكلي على سوق العراق للأوراق المالية

$$
\text { وخاصة للفترة بعد العام (2003). }
$$

- - أهمية البحث في بيان ومعرفة العوامل التي تترك أثراً كأسعار النفط والنمو الاقتصادي والاستثار الاجنبي وغيرها على مؤشرات السوق الملالي، وبالتالي قيام الحكومة بوضع السياسات المطلوبة لتحقيق الاستقرار في القطاع الخاص في تقليل العجز وجذب الاستثمار الاجنبي لتخفيف الضغط على القطاع الحكوي.

\section{1 مشكلة البحث}

ان سوق العراق للاوراق المالية والتي تأسست حديثا في عام (2004) ما زالت

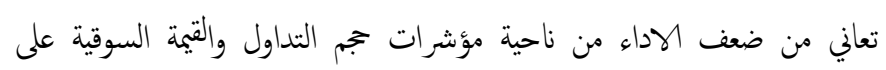

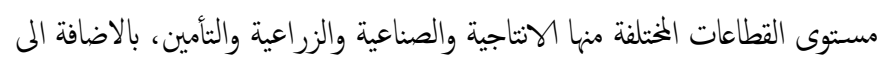
وجود تقلبات وانعدام الاستقرار في اداء السوق من ناحية حجم النشاط ودرجة السيولة (صيوان، 2016، 407) (الحكيم ودلول، 2016، 252)، وكذلك فان السوق تعاني من مشاكل متعلقة بالسياسات الاقتصادية والتي يدفع المستثمرين الى تجنب الدخول الى السوق الملالي والتي تؤثر بدورها سلباً على القطاع الملالي والاقتصاد الوطني (الموسوي واليساري والجنابي، 2018، 98)، فضلا عن أن مؤشرات السوق الملالي من ناحية رسملة السوق والقيمة المثداولة في العراق هي متدينة جدا مع مثيلاتها في الاسواق المالية في دول الخليج العربي أو ايران أوتركيا وغيرها. 


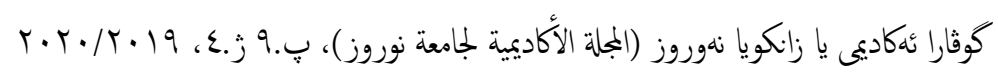

قياس حركة التغير في أسعار أسهم عينة من الشركات المساهمة التي تتداول أسهمها بانظام وتمثل قطاعات السوق مستخدما الرق القياسي المئوي (التقرير السنوي

$$
\text { العاشر، سوق العراق للأوراق المالية، 2013، 11). }
$$

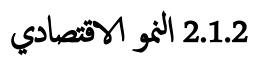

وهي النسبة المئوية السنوية لنمو الناتج المحلي الإجلالي بأسعار السوق على أساس العملة المحلية الثابتة، وأن إجلالي الناتج المحلي هو إجالي القيمة المضافة من قبل جميع المنتجين المقمين في الاقتصاد بالإضافة إلى ضرائب الانتاج مطرومًا منها أي إعانات

$$
\begin{aligned}
& \text { غير مدرجة في قيمة المنتج (قاعدة بيانات البنك الدولي). } \\
& \text { 3.1.2 سعر الصرف }
\end{aligned}
$$

يشير سعر الصرف الرسمي للعملة المحلية إلى سعر الصرف الذي تحدده السلطات من العملة الاجنبية أو سعر الصرف المحدد في سوق الصرف المصرح به قانونًا، حيث يتم حسابها كتوسط سنوي على أساس المتوسطات الشهرية (وحدات العملة المحلية بالنسبة للدولار الأمريكي)، أي أنه سعر العملة المحلية من العملة الأجنبية أي أنه يقيس سعر عملة واحدة في مقابل عملة أخرى(صندوق النقد الدولي).

\subsection{2 الاستثار الاجنبي المباشر}

يقصد بها صافي تدفقات الاستثمار للحصول على مصلحة إدارية دائة (10 في المائة أو أكثر من أسهم التصويت) في مؤسسة تعمل في اقتصاد دولة اخرى غير اقتصاد دولة المستثمر. هو مجموع رأس مال الأسهم وإعادة استثمار الأرباح ورأس المال طويل الأجل الآخرى ورأس المال قصير الأجل كما هو موضح في ميزان المدفوعات. والمؤشر هنا يقيس صافي الندفقات الداخلية (تدفقات الاستثمار الجديدة مطروحا منها اعادة الاستثمر ) مقسوما على إجلالي الناتج المحلي (UNCTAD, 2018).

\subsection{2 معدل الفائدة}

يشير معدل الفائدة المى العائد على رأس المال المستثمر خلال فتزة زمنية محددة عادة تكون سنة واحدة، إن الزيادة في أسعار الفائدة تزيد من تكلفة الفرصة البديلة للاحتفاظ بالمال وبذلك يحصل التبادل بين الاوراق المالية التي عليها فائدة ومن ثم يتم انخفاض أسعار الأسهم، وبالتالي فإن التغيير في أسعار الفائدة الوسمية يحرك أسعار الموجودات في الاتجاه المعاكس، والعلاقة السلبية ظهرت في العديد من الدراسات السابقة (Kandir, 2008)، وتم قياسه بمعدل الفائدة الحقيقي هو سعر
- - تقديم عدد من الاستناجات والمقترحات المى متخذ القرار الاقتصادي والمالي في العراق لوضع سياسات مالية ونقدية مشجعة لتطوير سوق العراق للاوراق المالية.

\section{1 فرضية البحث}

يتركز فرضية البحث على وجود تأثير معنوي لمتغيرات الاقتصاد الكلي المخسة والمتثلة في (سعر النفط، النمو الاقتصادي، سعر الصرف، نسبة الاستثمار الاجنبي المباشر المى اجلالي الناتج المحلي ومعدل الفائدة) مجتمعاً في المؤشر العام لسوق العراق للأوراق المالية للفترة (2005-2018).

\section{1 حدود البحث}

تتمثل الحدود الزمانية لهذا البحث للفتزة (2005-2018)، بجيث يتضمن سلسلة زمنية سنوية لمتغيرات اقتصادية متثثلة في (سعر النفط، النمو الاقتصادي، سعر الصرف، نسبة الاستثار الاجنبي المباشر الى اجلي الناج المحلي ومعدل الفائدة) كتغغرات مستقلة مع المؤشر العام للسوق المللي كتغغير معتمد، ويعتبر سوق العراق للاوراق المالية الحدود المكلية لهذا البحث.

\section{1}

تم استخدام ادوات مختلفة لاختبار الفرضيات من خلال الحزمة القياسية ( Eviews 8) بالاعتماد على منهجين يتمثل الوول في المنهج الوصفي لتطور متغيرات الاقتصاد الكلي ومؤشر السوق الملالي خلال فترة البحث، والمنهج الثاني يتمثل في التحليل الكمي من خلال الاساليب الاحصائية كلمتوسط والانحراف المعياري واختبار جاكبيرا والانحدار المتعدد بطريقة المربعات الصغرى لتحديد اثر متغيرات الاقتصاد الكلي على سوق العرق للآوراق المالية.

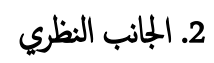

\section{2 تعاريف متغيرات البحث}

1.1.2 مؤشر السوق

إن مؤشر السوق هو رق قياسي مئوي يقيس حركة الاسهم المتداولة بشكل منتظم من قبل عنية من الشركات المدرجة في السوق الملالي، وارتفاع المؤشر يشير الى لي ارتفاع السوق بالكامل نتيجة تثثيل المؤشر للحركة العامة للسوق. إذ أن المؤشر العام

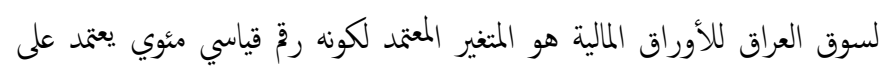




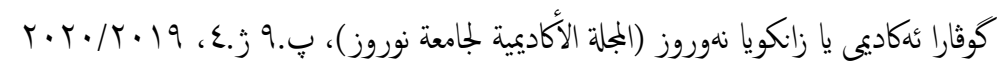

الشركات وتداول اوارقها المالية والرقابة على نشاط سوق التداول النظاي والموازي (باش والقريشي، 2017، 197)، وأيضاً بانشاء سوق العراق للاوراق المالية والتي تمثل مؤسسة ذات نقع عام لا تستهدف الربح في أعمالها وذات تمويل ذاتي ويتولى ادارتها بجلس المحافظين الذي يمثل متلف الشرائح الاقتصادية في الدولة، اول جلسة تداول باشرت في (2004/6/24) ضمن هيكة الاقتصاد العراقي والتوجه نحو السوق الحر، بجيث بلغ عدد الشركات المسجلة في أول جلسة (15) شركة مساهمة فقط بعضها كان مدرجاً في سوق بغداد للأوراق المالية المنحل. إن تطور هذا السوق بشكل ملحوظ واضح منذ افتتاحما فعلى سبيل المثال الانضام إلى الاتحاد الأوربي والآسيوي للأسواق المالية والتي تتكون من ثلاثين بورصة في العام (2005) ومن ثخ السمح لغير العراقيين بالتداول للأسهم بيعاً وشراءاً في العام (2007)، وتطوير آليات التداول باستخدام النظم الألكترونية في تداول الأسهم بدلا من اللوحات البلاستيكية منذ العام (2009) ودخولها عام (2010) في قاعدة بيانات صندوق النقد العربي للأسواق المالية العربية (Asaad, 2014A, 62-63). بشكل عام فان مؤشرات التداول في سوق العراق للأوراق المالية تتجه نحو الصعود كعدد الاسهم المتداولة، حجم النداول، والتمة السوقية والمؤشر العام للاسعار في السوق كما يوضحه الجدول (1)، حيث ارتغع عدد الاسهم المنداولة مقاساً بملايين الأسهم، حيث تداول (556391) مليون سهم للعام (2005) وبنسبة نمو بلغت (286\%) مقارنة مع العام (2004) بتداول (14393) مليون سهم، بينا بلغ تداول الأسهم (871182) مليون سهم للعام (2013)، أي بنسبة نمو حوالي (60\%) مقارنة مع العام (2004)، مع وجود نسب نمو مختلفة للأعوام الأخرى، وأيضاً إرتفاع مؤشر حجم التداول مقاساً كؤشر للسيولة ويقاس بملايين الدنانير العراقية، حيث بلغ (366809) مليون دينار اللعام (2005) وإرتغ إلى (284022) مليون دينار عام (2013)، ويلاحظ إرتفاع لمعدل نمو قيمة التداول خلال السنوات المّس الأولى من إفتتاح سوق العراق للأوراق المالية ومن ثم التراجع الحاد في العام (2009) و(2010)، والشكل (2) يوضح حركة واتجاهات هذه المؤشرات. إن هذا الصعود قد يرجع إلى سياسات وقرارات مجلس المحافظين الهادفة إلى تطوير البنية التحتية للسوق وازدادت ثقة المتعاملين بالسوق وقدرة السوق على جذب الاستثمرات والمدخرات Asaad, (المحلية وحتى الاستثمارات الخارجية بعد السماح لغير العراقيين للنداول فيها

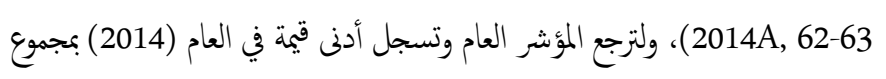

فائدة الإقراض المعدل للتضخم كما تم قياسه بواسطة معامل انكماش إجالي الناتج

$$
\text { الحلي (قاعدة بيانات البنك الدولي). }
$$

6.1.2 أسعار النفط

أسعار النفط هي تلك التي تشير ويقصد بها عادة إلى متوسط سعر استيراد النفط من منظمة التعاون الاقتصادي والتنمية (تأمين النكليف والشحن) حسب وكالة الطاقة الدولية الأمريكة. تقيس أسعار النفط الخام السعر الفوري لختلف براميل النفط، وعادة إما تكون غرب تكساس الوسيط أو مزيج برنت أو أحياناً يتم تحديد سعر سلة أوبك وسعر العقود الآجلة في البورصة ( EIA, Annual Energy .(Outlook 2011 2.2 يعتبر نظرية التسعير بالمرابحة (Arbitrage Pricing Theory) هي القاعدة الاساسية التي بنيت عليها هذا البحث، والتي أوضحت بان النموذج تصبح متوازنا لكونها تسمح بتحقيق التوازن في العائد على الموجودات بالاعتماد على عدة عوامل

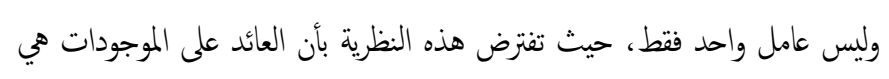
دالة خطية من عوامل الاقتصاد الكلي الخخلفة أو نظرياً مؤشرات السوق الملالي، وتنص النظرية على أن العائد المحقق على الموجودات يتكون من العائد المتوقع على ذلك الموجود في بداية الفترة الزمنية والادراك الغير متوقع لعوامل الخطر خلال تلك لك لك الفترة الزمنية بالإضافة إلى الخخاطر الخاصة بالشركة (Ross, 1976)، وجاءت هذه النظرية كبديل لنظرية تسعير الموجودات الرأسمالية (CAMEL) التي توضح العلاقة التوازنية بين العائد المطلوب على الموجودات والمخاطرة مع افتراض كون معامل بيتا يبقى ثابت، ويعني ذلك بأن نظرية تسعير الموجودات يحدد عائد الورقة المالية بعامل او متغير اقتصادي واحد فقط (Sharp, 1964).

\section{2 تطور سوق العراق للاوراق المالية}

بدأت محاولات العراق بانشاء سوق للاوراق المالية أبان الحكى الوطني عام (1921)، وبعد ذلك صدر قانون بورصة التجارة عام (1936)، وفي العام (1943) صدر قانون التجارة التي لم يرى النور الى حين صدور قانون رقٌ (24) للعام (1991) والذي عرف بسوق بغداد للأوراق المالية والتي استمر الى العام (2003)، وقرر سطلة الائتلاف المؤقتة بامر (74) للعام (2004) بالغاء القانون السابق وانشاء هيئة الاوراق المالية العراقية والتي تتولى سلطة الاشراف والرقابة على حركة تأسيس 


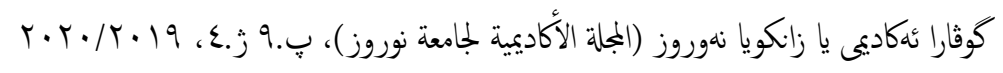

والتي جعلت الموضوع محل اهتمام من قبل الباحثين، والاتي بعض من هذه الدراسات:

هدفت دراسة باش والقريشي (2017) الى معرفة درجة تأثير متغيرات السياسة المالية وخاصة النقات العامة والضرائب على مؤشرات الاداء في السوق الملالي العراقي للفترة (2004-2014) باستخدام المنهج القياسي، والنتائج أوضحت بأن تأثير السياسة المالية كان ضعيفا في مؤشرات أداء السوق وكان مؤشر حجم التداول الاكثر تأثيرا بالسياسة المالية مقارنة مع بقية المؤشرات، وافترحت البحث بتقليل الفجوة الموجودة بين النفقات الجارية والاستثمرية والبدء بالاصلاح الضريبي في العراق مع فتح فروع اخرى لسوق العراق في مدن اخرى داخل البلد. بينا دراسة حسن وصباح في العام (2019) وجدت علاقة تكملية طويلة الأجل وقصيرة الاجل بين بعض مؤشرات الاقتصاد الكلي كمدل التضخم، عرض النقد، سعر الصرف ومعدل الفائدة مع مؤشر أسعار الهسم لسلسلة زمنية شهرية باستخدام التحليل القياسي للفتزة (2006-2015)، وكانت تأثير معدل التضخم وعرض النقد معنوي سلبي بينا تأثير معدل الفائدة معنوي ايجابي مع عدم معنوية سعر الصرف على مؤشر أسعار الاسهم، واقترحت البحث بضرورة خلق مناخ اقتصادي لتشجيع الاستقرار الاقتصادي ودع انشطة الشركات في سوق الاسهم. قام دراسة المعموري والزبيدي عام (2014) بالتعرف على أثر تغيرات سعر الصرف للدينار العراقي على المؤشر العام لأسعار أسهم الشركات المدرجة في سوق العراق للاوراق المالية للفتزة (2005-2011)، وأوضحت النتائُ بوجود علاقة تكملية مشتركة مع وجود علاقة سبية ثنائية الاتجاه بين متغيرات البحث، وكذلك استخدام مؤشر السوق كؤشر عام حول مسار النشاط الاقتصادي في المستقبل، وأيضاً اعتبرت البحث سعر الصرف أحد العوامل المؤثرة على أدء سوق الاسهم بوصفه أحد محددات النشاط الاقتصادي واحتالية استخداهما للتأثير في مؤشرات سوق العراق للآوراق المالية. في حين توصلت دراسة المرشدي عام (2018) الى وجود علاقة ارتباط وتأثير إيهابية ومعنوية بين مؤشرات سوق العراق للاوراق المالية وهي (عدد الاسهم المثداولة، ججم التداول، القيمة السوقية، مؤشر الاسعار وعدد الشركات المتداولة) والاستثار الاجنبي المباثر باستخدام طريقة المربعات الصغرى العادية للفتزة (2004-2015).
(92) نقطة فقط والتي قد يرجع ذلك الى العمليات الارهابية والوضع الامني المتذبذب ومن ثخ لتبدأ مرحلة الصعود لتسجل أرقام قياسية خلال المدة (2015-

.2018

جدول 1

مؤشرات سوق العراق للأوراق المالية للفترة (2004-2018)

\begin{tabular}{|c|c|c|c|c|}
\hline Year & $\begin{array}{c}\text { جم الثداول (مليون } \\
\text { دينار) }\end{array}$ & 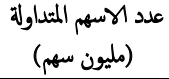 & القمة السوقية (مليون دينار) & مؤشر السوق \\
\hline & Volume Traded & Share Traded & Capitalization Market & Index ISX \\
\hline 2004 & 127950 & 143936 & 17155 & $\cdots$ \\
\hline 2005 & 366809 & 556391 & 2910 & 45.64 \\
\hline 2006 & 146891 & 579749 & 2764 & 25.29 \\
\hline 2007 & 427367 & 152990 & 2492 & 5934. \\
\hline 2008 & 301350 & 150853 & 2741 & 58.36 \\
\hline 2009 & 411928 & 211290 & 3125 & 100.86 \\
\hline 2010 & 400359 & 255659 & 3682 & 100.98 \\
\hline 2011 & 941198 & 49237 & 5552 & 136.3 \\
\hline 2012 & 893825 & 625639 & 5426 & 124.14 \\
\hline 2013 & 284022 & 871182 & 1324 & 113.15 \\
\hline 2014 & 898315 & 743852 & 1270 & 92 \\
\hline 2015 & 456179 & 579640 & 1054 & 730.56 \\
\hline 2016 & 426788 & 917542 & 9582 & 649.48 \\
\hline 2017 & 386879 & 631420 & 9729 & 580.54 \\
\hline 2018 & 232681 & 376806 & $\ldots$ & 510.12 \\
\hline
\end{tabular}

المصدر: الجدول من اعداد الباحثة بالاعتاد على التقارير السنوية لسوق العراق للأوراق المالية للفتزة (2004-2018).

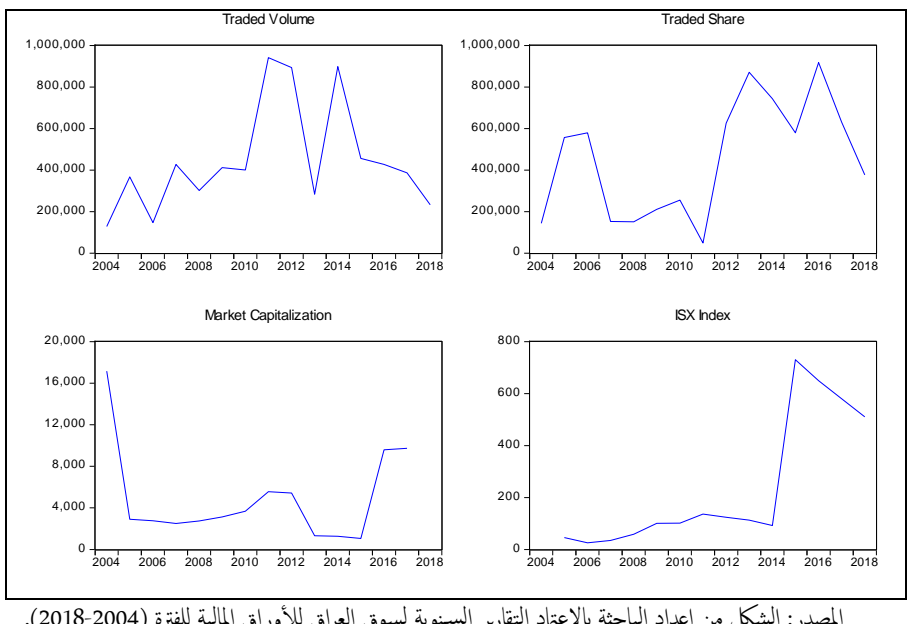

المصدر: الشكل من اعداد الباحثة بالاعتماد التقارير السنوية لسوق العراق للأوراق المالية للفترة (2004-2018).

الشكل 1. حركة مؤشرات سوق العراق للأوراق المالية للفترة (2004-2018).

4.2 الدراسات السابقة

يلاحظ عند مراجعة الدراسات السابقة عن الاسواق المالية وأداءها وتأثرها بالعوامل الاقتصادية المختلفة في العديد من الدول ولفترات زمنية متباينة وباستخدام أساليب احصائية وقياسية متنوعة، ولحد الان كانت النتائج غير حاسمة إلا أن الكثير من هذه الدراسات توصلت الى وجود صلة بين مؤشرات السوق المالي ومتغيرات الاقتصاد الكلي، ولذلك جاءت هذا البحث لتقليل الفجوة التي مازالت موجودة 


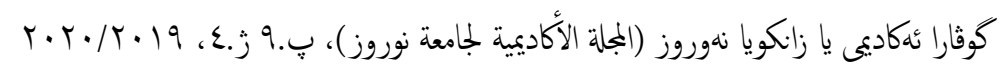

العلاقة السلبية طويلة الاجل مع سعر الصرف والاحتياطات الاجنية وأسعار الذهب ومعدل الفائدة مع عدم معنوية الاستثمار الاجنبي المباشر والناتج الصناعي والاستيراد والتصدير مع عائد الاسهم (Umer, 2016). بينا توصلت دراسة اخرى باستخدام نظرية التسعير بالمرابحة الى أن عرض النقد ومعدل التضخم تؤزر على عائد الاسهم في كنيا ويعتبر كمحددات معنوية للمدة (2013-2003)، ببنا جاءت تأثير سعر الصرف سلبي على عائد الاسهم ومعدل الفائدة لم يكن ذو أهمية في الثأثير (Ouma , 2014). بينا لوحظ العلاقة السلبية بين كل من عرض النقد ومعدل الفائدة ومعدل التضخم مع المؤشر العام لسوق الاسهم الماليزية (Asaad, 2017). يتيين من استعراض الدراسات السابقة بانه مازالت هناك جفوة بكثية في اختبار

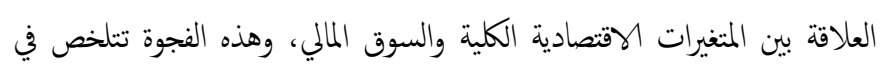
عدم وجود دراسات عديدة تركز على الدول النامية مثل العراق، وان وجدت فانها لم تدرس السوق المالي في العراق بشكل تطبيقي مع المتغيرات الاخرى، وقد يرجع ذلك الى عدم توفر بيانات لفترة زمنية بسبب حداثة هذا السوق. هناك دراسات عديدة حول العلاقة والثأثير بين العوامل الاقتصادية الكلية والسوق المالي اله انه لحد الان لم يقدم دليل محدد متفق عليه حول اتجاه العلاقة بينها، وهذا يعطي فرصة أمام الباحثين في التخصص بإجراء المزيد من الدراسات عن الموضوع، ولتحقيق ذلك جاءت هذا البحث لفحص الموضوع تطبيقيا لبعض المحددات الاقتصادية للسوق الملالي في العراق. حيث يعتبر العراق بيئة البحث الحالية والتي تختلف عن الدراسات السابقة لكونها بيئة انفتحت على العالم لفترة لم يتجاوز العقدين من الزمن، وبالتالي فان ننائج هذا البحث ستكون مختلفا باختلاف المنهجية والنطاق الزمني لها ومعرفة بعض الاسباب التي جعلت سوق العراق للاوراق المالية ضعيفة على الرغز من استخداما للنظام الككتروني وليست جاذبة على الاطلاق للاستثار ، في حين تتشابه البحث مع مثيلاتها في ربط السوق الملالي بعض المتغيرات الاقتصادية الكلية كمحددات لها.

\section{3. الجانب التطبيقي للدراسة}

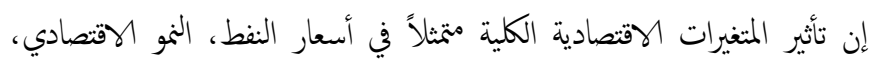
وسعر الصرف للدينار العراقي مقابل الدولار الامريك، نسبة تدفقات الاستثار الاجنبي المباثر الى اجالي الناتج المحلي وأسعار الفائدة على المؤشر العام لسوق
ان دراسة الموسوي واخرون (2018) قامت بتحليل دور عزز الموازنة الحكومية في النغيرات الحاصلة في مؤشرات سوق العراق للأوراق المالية للمدة (2005-2016) باستخدام طريقة الانحدار الخطي البسيط، وأقرت ننائُ البحث بوجود علاقة ارتباط ذات دلالة احصائية بين المتغيرين والتي تدل على وجود امتداد للسوق الملالي في العراق الى السياسة الاقتصادية المتبعة في البلد. بينا تناولت دراسة الدعمي (2012) اثر السياسة النقدية على مؤشرات النداول لسوق نيويورك للاوراق المالية للمدة (1990-2010) باستخدام نموذج كويل للتخلف الزمني، وجاءت النتائُ متطابقة مع منطق النظرية الاقتصادية بمعنوية العلاقة الطردية بين عرض النقود وسعر الفائدة مع مؤشرات السوق الملالي، واقترح البحث امكانية استخدام السياسة النقدية في الثاثير على النشاط الاقتصاد من خلال التأثير في مؤشرات السوق الملالي وبالتالي توجيه السياسة النقدية بالاتجاه الصحيح. فيا هدفت دراسة الحكيم ودلول (2016) الى مقارنة سوق العراق للاوراق المالية قبل وبعد استخدام نظام التداول الاكتروني للمدة (2005-2013)، والنتائج أوضحت بوجود تحسن واضح في تداول مؤشرات السوق، واقترح البحث بربط سوق العراق للاوراق المالية ببقية الاسواق العربية وخاصة المجاورة لتشجيع عملية تحويل الاموال وزيادة السيولة. وهناك دراسة اخرى أجريت في فلسطين للبيانات الشهرية للفترة (2011-2017) وجدت بأن مؤشر أسعار المستهاك لقياس التضخم وسعر الصرف والناتج الحلي الاجالي يؤثر سلبياً على السوق الفلسطيني بينا لم يجد أي تأثير لكل من مؤشر الانتاج الصناعي والميزان التجاري على السوق المالي ( Jabarin, Nour \& .(Atout, 2019 أما دراسة Ilahi, Ali \& Jamil في العام (2015) ربطت بين بعض متغيرات الاقتصاد الكلي كمعدل التضخم، سعر الصرف ومعدل الفائدة ومؤشرسوق كراشي المالية لتمثيل لعائد سوق الاسهم للمدة كانون الثاني 2007 المى تشرين الاول 2012 باستخدام الانحدار الخطي المتعدد، وأوجدت النتائج بعدم معنوية الثأثير للمتغيرات على عائد السوق والتي تشير الى ضعف العلاقة بين متغيرات البحث، وهذه النتأُج جاءت متوافقة مع نتابُج دراسة اخرى بكون معدل التضخم ومعدل الفائدة غير معنوية في سنغافورة (Khan, Khan \& Rukh., 2012; Lee \& Wang, 2012)، ودراسة اخرى في باكستان للمدة (2005-2015) وجدت علاقة ايجابية طويلة الاجل بين مؤشر الاسهم مع معدل التضخم وعرض النقد وأسعار النفط، فياكانت 


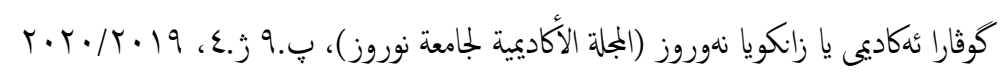

Estimation Equation: ISXI $=\mathrm{C}(1)^{*} \mathrm{OP}+\mathrm{C}(2)^{*} \mathrm{EG}+\mathrm{C}(3)^{*} \mathrm{ER}+$ $\mathrm{C}(4)^{*} \mathrm{FDI} \_\mathrm{GDP}+\mathrm{C}(5)^{*} \mathrm{IR}+\mathrm{C}(6)+\mathrm{e}$

حيث ان = المؤشر العام لسوق العراق للاوراق المالية. ISXI معامل سعر النفط. = OP EG = Eعامل النمو الاقتصادي.

معامل سعر صرف الدينار العراقي مقابل الدولار الامريكي. ER FDI_GDP = معامل نسبة الاستثمار الاجنبي المباشر الى اجالي الناتج المحلي. معامل معدل الفائدة. IR

C(6) Cابت المعادلة ( قيمة المتغير التابع في حالة كون المتغيرات المستغلة مساوية الصفر). e يوضح الجدول (3) بيانات السلسلة الزمنية السنوية للمتغيرات المستقلة والمتغير المعتمد في جمهورية العراق، حيث قبل البدء باستخدام التحليل لغرض اختبار الفرضيات تم القيام ببعض الاختبارات للتعرف على طبيعة البيانات كعرض البيانات في رسومات بيانية والتي تشمل (14) مشاهدة لمعرفة حركة المنغيرات كما في الشكل

جدول 3

مشاهدات متغيرات البحث للفترة (2018-2005)

\begin{tabular}{ccccccc}
\hline \hline Year & ISXI & OP & EG & ER & FDI_GDP & IR \\
\hline 2005 & 45.64 & 54.57 & 4.40 & 1472 & 1.03 & 6.56 \\
2006 & 25.29 & 65.16 & 10.16 & 1467.42 & 0.59 & 6.62 \\
2007 & 34.59 & 72.44 & 1.38 & 1254.57 & 1.09 & 10.43 \\
2008 & 58.36 & 96.94 & 8.23 & 1193.08 & 1.41 & 10.54 \\
2009 & 100.86 & 61.74 & 3.38 & 1170 & 1.43 & 7.82 \\
2010 & 100.98 & 79.61 & 6.4 & 1170 & 1.01 & 6.1 \\
2011 & 136.3 & 111.26 & 7.55 & 1170 & 1.12 & 6 \\
2012 & 124.14 & 111.63 & 13.94 & 1166.17 & 1.56 & 6 \\
2013 & 113.15 & 108.56 & 7.6 & 1166 & -1 & 6 \\
2014 & 92 & 98.97 & 0.7 & 1166 & -4.34 & 6 \\
2015 & 730.56 & 52.32 & 2.48 & 1167.33 & -4.27 & 6 \\
2016 & 649.48 & 43.64 & 13.57 & 1182 & -3.68 & 6 \\
2017 & 580.54 & 54.13 & -1.67 & 1184 & -2.61 & 4 \\
2018 & 510.12 & 64.9 & 0.63 & 1182.75 & -2.16 & 4 \\
\hline \hline
\end{tabular}

المصدر: الجدول من اعداد الباحثة بالاعتاد على

The Global Economy, Business and Economic Database, https://www.theglobal economy.com/

Central Bank of Iraq. (2011). Annual Report 2004-2019, Statistics and Research Department, Iraq. Available at http://www.cbi.iq

UNICTAD. World Investment Report Series, 2004-2019. United Nations Conference on Trade and Development http://unctad.org/en/Pages/Publication/

WorldInvestmentReports(1991-2009).aspx.
العراق للاوراق المالية للفترة الممتدة (2005-2018) هي الهدف الهاسي لهذا البحث، ويتضمن هذا المبحث التوصيف الاحصائي لبيانات البحث مع تحليل النتائج، حيث يعتبر المؤشر العام لسوق العراق للاوراق المالية المتغير المعتمد بينا يمثل متغيرات الاقتصاد الكلي المتغيرات المستقلة لهذا البحث، بعد أن تم تناول

$$
\text { الجانب النظري والدراسات السابقة في المبحث الاول. }
$$

1.3 مصادر البيانات

إن سوق العراق للاوراق المالية يمثل عينة لمجتع دول الشرق الاوسط للخلفية التي اكتسبتها العراق بعد انتاحما الاقتصادي على العالم وخاصة بعد نيسان (2003)، حيث عاش العراق حالة من الانتاح الواسع وظهور السوق العراقي كنافس للادول العربية بشكل عام ودول الجوار بشكل خاص ولاسيا بعد التطورات الحاصلة في سوق العراق للاوراق المالية والنذبذب المستمر في حركة تدفقات الاستثار الاجنبي المباشر والنمو الاقتصادي وغيرها من متغيرات الاقتصاد الكلي. إذ أن بيانات متغيرات الاقتصاد الكلي والمؤشر العام لسوق العراق للأوراق المالية تح جمها من المصادر الاولية مختلفة ومن المصادر الثانوية كالتقارير والنشرات السنوية المحلية والدولية الصادرة من الجهات العربية الاجنبية لمتغيرات البحث للفتزة (-2005

2018)، وكما هي موضح في الجدول (2). جدول 2 مصادر البيانات لمتغيرات البحث

\begin{tabular}{|c|c|c|}
\hline المصدر & نوع المتغير & \\
\hline التقارير السنوية لسوق العراق للأوراق المالية & المؤشر العام لسوق العراق & المتغير \\
\hline لفلفترة (2004-2018) & للاوراق المالية & المتمد \\
\hline 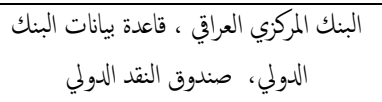 & متغيرات الاقتصاد الكلي للدراسة & المستقل - المتغير \\
\hline
\end{tabular}

2.3 توصيف النموذج

إن هذا البحث ركز على تأثير خمسة متغيرات مستقلة متثلة في متغيرات الاقتصاد الكلمي كسر النفط، النمو الاقتصادي، سعر الصرف، نسبة الاستثمار الاجنبي المباشر الى اجالي الناتج المحلي ومعدل الفائدة على المتغير المعتمد المتمثل في المؤشر العام لسوق العراق للاوراق المالية باستخدام طريقة المربعات الصغرى ( Ordinary Least Square )، ويأتي استخدام هذا النموذج نتيجة عدم توفر بيانات سلسلة زمنية طويلة عن السوق المالي في العراق لكونها تأسس حديثاً. 


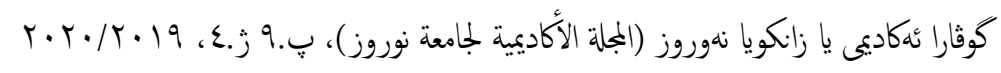

البحث في العام (2018) وكان أدنى مستوى لأسعار النفط في العام (2016) عندما بلغت (43.64) دولار للبرميل. إن النغير في الناتج المحلي الاججلي في العراق قد كان متذبذباً خلال فترة البحث وكان أدنى قيمة في العام (2017) وكان قد سجل أعلى قيمة لها في العام (2012) مقارنة مع الاعوام السابقة، وكان متوسط النمو السنوي في العراق من العام (2005) الى العام (2018)كان بمعدل (4.5\%). إن تغير العملة العراقية بعد (2003) جاءت نتيجة توجه النظام الجديد الى اقتصاد السوق، حيث كان سعر الصرف للدينار العراقي في العام (2005) بلغت (1472) مقابل دولار واحد، ومن ثم بدأت ارتفاع في سعر الصرف مقارنة مع السنة السابقة، واستمرت هذه الارتفاع الى نهاية (2018) والتي تميزت خلال فترة البحث ولا سيا بعد العام (2009) بنوع من الاستقرار والثبات في سعر الصرف والتي قد يرجع لربما لتدخل البنك المركزي في مزايدات العملة. إن نسبة تدفقات الاستثار الاجنبي المباشر الى الناتج المحلي الاجالي في العراق للفترة (2005-2018) هي لم تصل الى (2\%) وهي نسبة قليلة جداً، في حين يكون هذه النسبة يتجاوز (10) في دول نامية صغيرة جداً، وحتى وقد وصلت هذه النسبة في العراق الى معدلات سلبية في

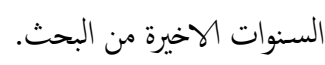
اما فيا يتعلق بمعدل الفائدة في العراق للعام (2004) بلغ (6.56\%) ثم شهد ارتفاعات مستمرة الى العام (2009)، ومن ثم بدأت بالتوجه نحو الاستقرار والثبات الى العام (2016) والتي بدأت بالانخفاض الى أربعة بالمئة فقط خلال السنتين الاخيتين لفترة البحث. ويستنتج من التوصيف السابق لمتغيرات الاقتصاد الكلي والمؤشر العام لسوق العراق للاوراق المالية بوجود تذبذب نحو الصعود والهبوط خلال فترة البحث، وهذا قد يرجع الى عوامل عديدة منها على سبيل المثال لا الحصر نتيجة توجه العراق الى اقتصاد السوق وتحديد الاسعار حسب قوانين

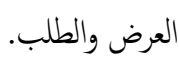
إن الجدول (4) يقدم توصيفاً لخصائص متغيرات البحث والتي تتضمن المتوسط، الانخراف المعياري، الحد الاعلى والحد الادنى، معامل الالتواء ومعامل التفلطح ومع اختبارات احصائية جاك بيرا لمعرفة احتالية التوزيع الطبيعي لبيانات البحث، حيث تبينت بان بيانات البحث كلها تتبع التوزيع الطبيعي نتيجة لعدم معنوية القيمة، وهذا يعني بعدم وجود اي عشوائية في البيانات، حيث ان متوسط المؤشر العام لسوق

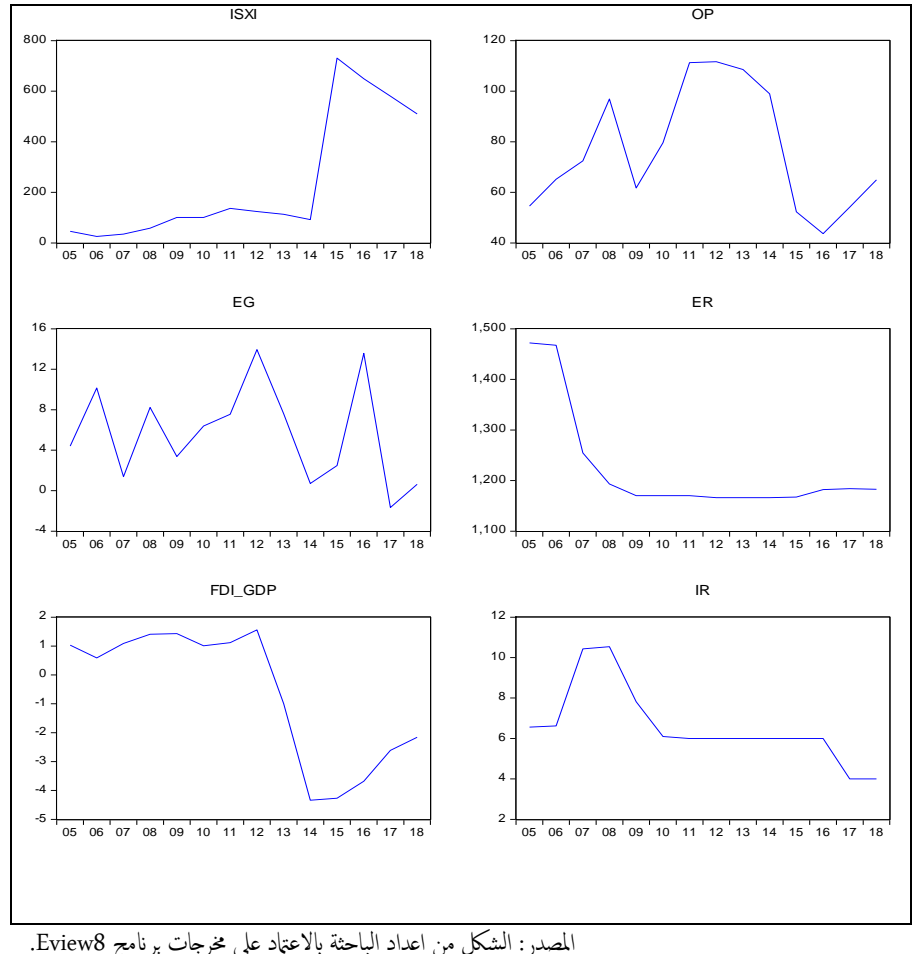

شكل 2. حركة متغيرات البحث للفترة (2018-2005) ان المؤشر العام لسوق العراق للاوراق المالية للفترة (2018-2005) موضح في الجدول (3) والشكل (2) بأن هذا المؤشر للعام (2005) بلغت (45) نقطة بعد ان تغير النظام الاقتصادي المى اقتصاد السوق وبدأت باستقبال الاستثرات الاجنبية المباشرة وتم تحويل سوق بغداد المى سوق العراق للاوراق المالية وقد ازداد المؤشر العام ليسجل (730) نقطة في العام (2015) اي بمعدلات مرتفعة جدا معارنة مع بداية سوق العراق للاوراق المالية. وكانت تطور هذا السوق من خلال ارتفاع مؤشراتها الرئيسية والقطاعية نتيجة وجود تغيرات جذرية في سياسة السوق سواء بالسماح بدخول المستثمرين الاجانب في عمليات البيع والشراء للاسهم واززياد عدد الشركات المدرجة وزيادة عدد الاسهم ورأسمالها، بينا كانت هذه الاستثمارات متوقةة لأكثر من عقدين نتيجة الحروب والحصار وعقيدة نظام الحكم السابق لاعتبار الاستثمر الاجنبي تدخل واضح في استقلالية القرارات وسياسة الدولة العراقية. إن العراق كدولة مصدرة للنفط قد استفادت من العام (2011) عندما وصلت أسعار النفط الى مستويات قياسية بحيث تجاوزت المئة دولار للبرميل الواحد كما هي موضح في الجدول (3) والشكل (2) خلال فترة البحث، أي بزيادة بلغت الضعف مقارنة مع العام (2005) حيث كانت (54.57) دولار للبرميل، واستمرت هذه الزيادات الى العام (2014) والتي بدأت مسارها نخو الانخفاض الى نهاية فترة 


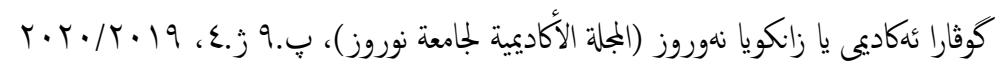

النمو الاقتصادي، سعر الصرف، نسبة الاستثمار الاجنبي المباشر الى اجلي الناتج

المحلي ومعدل الفائدة) في المؤشر العام لسوق العراق للاوراق المالية كتنغر معتمد في

البحث للفترة الممتدة (2005-2018)، ويعرض الجدول (5) تأثير المتغيرات المستقلة

المتثثلة في كل من معدل التضخم، معدل الفائدة وسعر الصرف للدينار العراقي مقابل

الدولار الامريكي في المتغر المعتمد المتمثل في سوق العراق للاوراق المالية.

جدول 5

تحليل الونحدار لمتغيرات البحث للفترة (2018-2005)

\begin{tabular}{|c|c|c|c|c|}
\hline \multicolumn{5}{|c|}{ Dependent Variable: ISXI } \\
\hline \multicolumn{5}{|c|}{ Method: Least Squares } \\
\hline \multicolumn{5}{|c|}{ Date: $10 / 03 / 19$ Time: 06:42 } \\
\hline \multicolumn{5}{|c|}{ Sample: 20052018} \\
\hline \multicolumn{5}{|c|}{ Included observations: 14} \\
\hline Variable & Coefficient & Std. Error & $\mathrm{t}$-Statistic & Prob. \\
\hline $\mathrm{OP}$ & -6.479063 & 1.831983 & -3.536639 & 0.0077 \\
\hline EG & 8.943304 & 7.824023 & 1.143057 & 0.2861 \\
\hline ER & -1.051299 & 0.397628 & -2.643924 & 0.0295 \\
\hline FDI_GDP & -38.03557 & 21.26494 & -1.788652 & 0.1115 \\
\hline IR & -30.75941 & 21.39334 & -1.437803 & 0.1884 \\
\hline $\mathrm{C}$ & 2146.715 & 599.3092 & 3.581983 & 0.0072 \\
\hline \multicolumn{2}{|c|}{ R-squared 0.851282} & \multicolumn{3}{|c|}{ Mean dependent var235.8579 } \\
\hline \multicolumn{2}{|c|}{ Adjusted R-squared0.758333 } & \multicolumn{3}{|c|}{ S.D. dependent var256.7454 } \\
\hline \multicolumn{2}{|c|}{ S.E. of regression 126.2152} & \multicolumn{3}{|c|}{ Akaike info criterion 12.81138} \\
\hline \multicolumn{2}{|c|}{ Sum squared resid 127442.2} & \multicolumn{3}{|c|}{ Schwarz criterion 13.08526} \\
\hline \multicolumn{2}{|c|}{ Log likelihood-83.67966 } & \multicolumn{3}{|c|}{ Hannan-Quinn criter. 12.78603} \\
\hline \multicolumn{2}{|c|}{ F-statistic 9.158590} & \multicolumn{3}{|c|}{ Durbin-Watson stat2.452402 } \\
\hline \multicolumn{2}{|c|}{ Prob(F-statistic)0.003651 } & & & \\
\hline
\end{tabular}

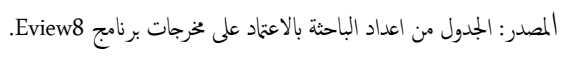

ISXI $=-6.47906297453^{*} \mathrm{OP}+8.9433044205^{*} \mathrm{EG}-$

\section{$1.05129913517^{*} \mathrm{ER} \quad$ - $\quad 38.035570679 * \mathrm{FDI}$ GDP}

$30.7594108665^{*} \mathrm{IR}+2146.715385861 \ldots \ldots .1$

إن الجدول (5) يوضح معامل التحديد في التغيرات الحاصلة في المؤشر العام لسوق العراق للاوراق المالية الى سعر النفط، النمو الاقتصادي، سعر الصرف، نسبة الاستثمار الاجنبي المباشر الى اجالي الناتج المحلي ومعدل الفائدة بنسبة (85\%)، بينما النسبة المتبقية يعود الى متغبرات أخرى لم يدخل في نموذج البحث. وكان اختبار النموذج معنوياً من خلال اختبار (F)، في حين كان سعر النفط ذو علاقة سلبية معنوية وجاءت العلاقة الايجابية غير المعنوية للمو الاقتصادي مع المؤشر العام لسوق العراق للاوراق المالية بشكل متطابق مع نتائج دراسة (Umer, 2016). بينا
جدول 4

التوصيف الاحصائي لمتغيرات البحث للفترة (2018-2005)

\begin{tabular}{ccccccc}
\hline \hline & ISXI & OP & EG & ER & FDI_GDP & IR \\
\hline Mean & 235.8579 & 76.84786 & 5.625000 & 1222.237 & -0.630000 & 6.576429 \\
Median & 107.0650 & 68.80000 & 5.400000 & 1176.000 & 0.800000 & 6.000000 \\
Maximum & 730.5600 & 111.6300 & 13.94000 & 1472.000 & 1.560000 & 10.54000 \\
Minimum & 25.29000 & 43.64000 & -1.670000 & 1166.000 & -4.340000 & 4.000000 \\
Std. Dev. & 256.7454 & 24.08987 & 4.841295 & 107.3104 & 2.301675 & 1.913618 \\
Skewness & 0.996780 & 0.300844 & 0.316290 & 1.874715 & -0.589698 & 0.979153 \\
Kurtosis & 2.219441 & 1.589544 & 2.074402 & 4.732470 & 1.670371 & 3.450536 \\
& & & & & & \\
Jarque-Bera & 2.673739 & 1.371659 & 0.733186 & 9.951476 & 1.842685 & 2.355469 \\
Probability & 0.262667 & 0.503672 & 0.693092 & 210.6903 & 0.397984 & 0.307976 \\
& & & & & & \\
Sum & 3302.010 & 1075.870 & 78.75000 & 17111.32 & -8.820000 & 92.07000 \\
Sum Sq. & & & & & & \\
Dev. & 856936.3 & 7544.181 & 304.6958 & 149701.7 & 68.87020 & 47.60512 \\
& & & & & &
\end{tabular}

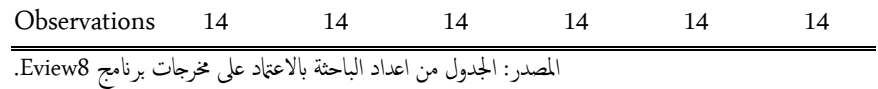
العراق للاوراق المالية (235.8579) وبانحراف معياري (256.7454)، ومتوسط أسعار النفط (76.84786) وبانحراف معياري (24.08987)، ومتوسط النمو الاقتصادي (5.625) وبانحراف معياري (4.841295)، وان متوسط سعر الصرف الدينار العراقي (1222.237 ) وبانحراف معياري (107.3104)، ومتوسط نسبة الاستثمر الاجنبي المباشر الى الناتج المحلي الاجلالي هو (0.63-) وبانحراف معياري (2.301675)، ومتوسط سعر الفائدة (6.576429) وبانحراف معياري (20.61796 )، ومتوسط معدل الفائدة (7.758750 ) وبانحراف معياري (1.913618). يستنتج من البيانات وسلوكها بان الهخراف المعياري يشير الى وجود تغيرات وتحركات اكبر لكل المؤشر العام لسوق العراق للاوراق المالية وسعر الصرف للدينار العراقي مقابل الدولار الامريكي وأسعار النفط الدولية وبقيم (6256.7454) و (107.3104) و (24.08987) على التوالي مقارنة مع كل من النمو الاقتصادي ونسبة الاستثمار الاجنبي المباشر الم اجالي الناتج المحلي ومعدل الفائدة وبتيم (4.841295) و(2.301675) و (1.913618)، وبالتالي يلاحظ بان معدل الفائدة هو الأقل تذبذباً مقارنة مع المتغيرات الاخرى بينما المؤشر العام لسوق العراق للاورق المالية الاكثر تذبذباً مقارنة مع متغيرات البحث.

3.3 تقدير النموذج

إن المعادلة (1) يوضح اثر متغيرات الاقتصاد الكلي الحمسة والمتمثلة في (سعر النفط، 


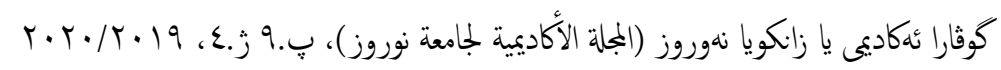

أقل من قيمة (2) ويعني بعدم وجود التعدد الخطي وبالتالي عدم وجود (VIF) الارتباط بين المتغيرات المستقلة، وفي الاخير نتائج اختبار احصائية رامسي توكد بعدم وجود مشكلة ادخال متغيرات غير صحيحة الهيا او مشكلة عدم تحويل البيانات كما في الجدول (9)، وتعتبر ننائُ هذه الاختبارات مرغوبة نتيجة تحقيقها لافتراضات نموذج الانحدار المتعدد بالشكل الصحيح.

$$
\text { جدول } 6
$$

Breusch-Godfrey Serial Correlation LM Test

Breusch-Godfrey Serial Correlation LM Test:

F-statistic1.018646

Prob. F(2,6)0.4160

Obs*R-squared3.548718 Prob. Chi-Square(2)0.1696

المصدر: الجدول من اعداد الباحثة بالاعتمد على مخرجات برنامج Eview8.

$$
\text { جدول } 7
$$

Correlogram of Residuals Test

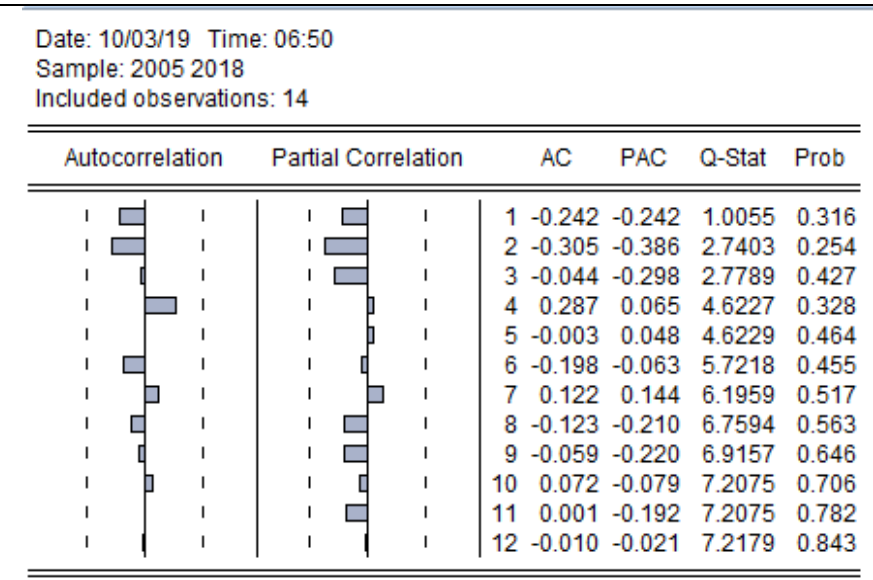

المصدر: الجدول من اعداد الباحثة بالاعتاد على خخرجات برنامج Eview8.

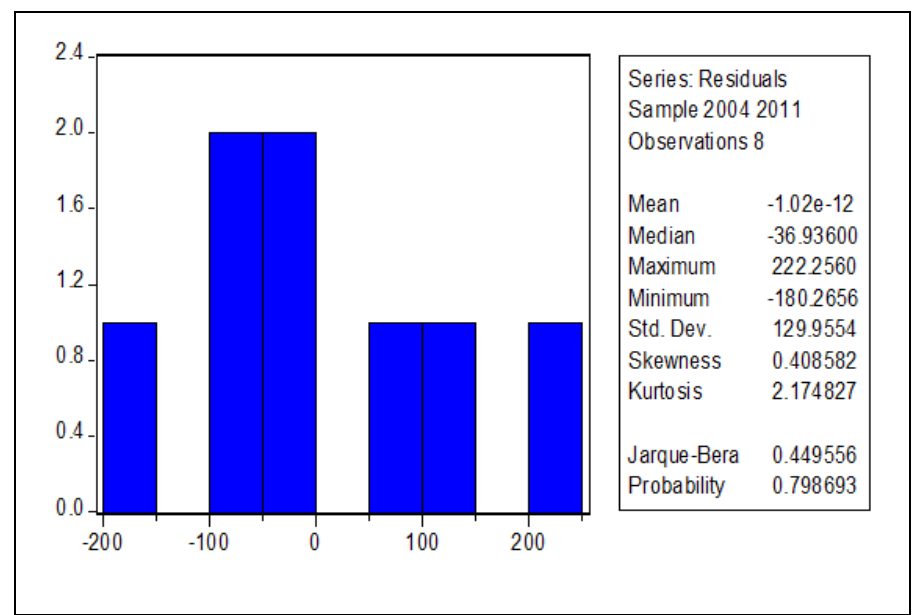

المصدر: الشكل من اعداد الباحثة بالاعتماد على تخرجات برنامج Eview8.

شكل 3.
كانت نتائج البحث هي سلبية ومعنوية سعر الصرف مع مؤشر السوق المالي والتي تتطابق مع النظرية الاقتصادية وبعض الدراسات السابقة في أن الارتفاع في سعر الصرف يؤدي الى انخفاض المؤشر العام للاوراق المالية (Ouma, 2016) وبعكس ما توصلت اليه نتاجُج دراسات اخرى (حسن وصباح، 2018: المعموري والزبيدي، ،(2014 بينا أظهرت نتائج البحث بعدم معنوية التأثير السلبي لنسبة الاستثمار الاجنبي المباشر الى اجلالي الناتج المحلي مع المؤشر العام للسوق الملالي بشكل متطابق مع ما توصل اليه (Umer, 2016) وعكس ما توصلت اليه دراسة (المرشدي، 2018)، والبحث الحالي وجدت العلاقة السلبية غير معنوية لمعدل الفائدة مع المؤشر العام Asaad, 2017; Umer, ) للسوق الملالي في العراق والتي تتوافق مع نتاجُج دراسات 2016)، وبشكل أو أخر جاءت متعارضة مع درسات اخرى ( Illahi \& et.al, Khan, et.al, Lee \& Mang, 2012 \& Ouma, 2016

$$
\text { بعد اختبار الانحدار المتعدد لمتغيرات البحث، 2019). }
$$

أ. معامل التحديد ومعنوية النموذج: أن عملية تقييم النموذج كان من خلال اختبار معامل R2 والذي بلغ (85\%)، ويشير ذلك الى أن النغيير في المؤشر العام لسوق العراق للاوراق المالية يرجع المى المتغيرات المستقلة معاً والمتمثلة في سعر النفط، النمو الاقتصادي، سعر الصرف، نسبة الاستثار الاجنبي المباشر الى اجالي الناتج المحلي ومعدل الفائدة، وأن النسبة المتبقية ترجع الى متغبرات اخرى (البواقي) التي لم تدخل في نموذج البحث. وأيضاً يوضح الجدول (5) بمعنوية النموذج أي أن جميع المتغيرات المستقلة معاً يفسر الثأثير على المتغير

$$
\text { المعتمد سوق العراق للاوراق المالية، وهذه الحالة مرغوبة. }
$$

ب. تحليل البواقي: إن الجدول (6) (7) يوضح بأن النموذج لا يواجه مشكلة Breusch-Godfrey Serial ) الارتباط الذاتي للبواقي باستخدام اختبار ، (Correlogram of Residuals Test) واختبار (correlation LM بينا الشكل (3) يوضح بأن البواقي يتبع التوزيع الطبيعي وذلك باستخدام اختبار (Histogram- Normaily test) وباختبار احصائية جاك بيرا، وان البواقي تتبع التوزيع الطبيعي وذلك لكون احتالية احصائية جالك بيراكان اقل من (5\%)، في حين أن الجدول (8) يبين بأن قيم معامل التضخم 


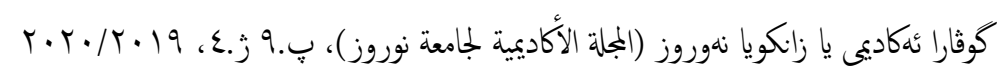

بينا جاءت عدم معنوية العلاقة الايجابية بين النمو الاقتصادي والسوق المالي

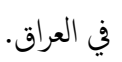

اوضحت الدراسات السابقة وجود علاقة وأثر بشكل ايجابي أو سلبي لسعر الفائدة ونسبة تدفقات الاستثمار الاجنبي المباشر الى اجلي الناجت المحلي في الدول المتقدمة والنامية على حد سواء، الا ان هذا البحث لم يثبت معنوية الثأثير السلبي لسعر الفائدة ونسبة الندققات للاستثمار الاجنبي الى الناتج المحلي على المؤشر العام للسوق العراقي.

ان نموذج البحث حققت الفرضيات الاحصائية والقياسية المرغوبة من معنوية النموذج ومعامل التحديد وعدم وجود مشكلة الارتباط الذاتي وثبات التباين

$$
\begin{aligned}
& \\
& \text { والمتوزيع الطبيعي للبواقي. }
\end{aligned}
$$

تحديث وتحسين سوق العراق للاوراق المالية وادخال الانظمة والتكنولوجيا الحديثة لغرض تفعيل دورها في الوساطة المالية بين وحدات العجز ووحدات

$$
\text { الفائض وتحقيق كفاءة السوق الملالي في اطار العولمة. }
$$

أهمية تفعيل دور السياسة المالية والنقدية في العراق من خلال تحديث النظام الضريبي وتقليل العتماد على النفط كمورد رئسي لتمويل موازنة العراق، وأيضاً قيام الحكومة بتوفير المناخ المناسب لدعز وانشاء الشركات المساهمة لدع الاقتصاد المحلي ووضع الخطط المناسبة لتخفيف عبء الديون الداخلية والخارجية. أهمية القيام بتفعيل قوانين الاستثمار وخاصة تجاه الاستثرار الاجنبي المباشر لجذب روؤس الاموال الاجنية من خلال توفير الاستقرار الامني والسياسي وتقليل الروتين والعمل على تقليل الفساد المالي والاداري والقضاء

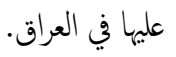
يقتح البحث بنتح فروع لسوق العراق في مدن اخرى مع تفعيل فرع السوق في مدينة أربيل عاصمة اقليم كردستان العراق.

هقتح البحث باجراء دراسات مستقلية بادخال متغيرات اقتصادية وغير اقتصادية اخرى من أجل تطوير نموذج البحث كمتغرات سياسية،
جدول 8

اختبار قيم معامل التضخم

\begin{tabular}{|c|c|}
\hline & Centered \\
\hline Variable & VIF \\
\hline OP & 1.589395 \\
\hline EG & 1.170856 \\
\hline ER & 1.485793 \\
\hline FDI_GDP & 1.954953 \\
\hline IR & 1.367690 \\
\hline $\mathrm{C}$ & NA \\
\hline
\end{tabular}

Included observations: 14

المصدر: الجدول من اعداد الباحثة بالاعتاد على مخرجات برنامج Eview8.

$$
\text { جدول } 9
$$

Ramsey RESET Test

Equation: EQ01

Specification: ISXI OP EG ER FDI_GDP IR C

Omitted Variables: Squares of fitted values

\begin{tabular}{lccc}
\hline & Value & $\mathrm{df}$ & Probability \\
\hline t-statistic & 3.388152 & 7 & 0.0116 \\
F-statistic & 11.47957 & $(1,7)$ & 0.0116 \\
Likelihood ratio & 13.59058 & 1 & 0.0002 \\
\hline \hline
\end{tabular}

المصدر: الجدول من اعداد الباحثة بالاعتماد على مخرجات برنامج Eview8.

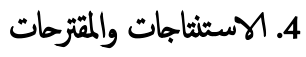

بعد عرض نتائُ البحث في المبحث الثاني، فان هذا المبحث يقدم بمهوعة من الاستنتاجات والمقتزحات وبالشكل الاتي:

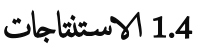

تبين بأن هناك تطور كير لسوق العراق للاوراق المالية خلال توصيف بيانات البحث للفتزة (2005-2018)، فقط سجل المؤشر العام للسوق (730) و (510) نقطة في العام (2015) و (2018) على التوالي مقارنة مع المؤشر الذي سجل (45.64) نقطة في العام (2005). اظهرت نتائج الدراساة الحالية العلاقة العكسية المعنوية بين سعر النفط وسعر الصرف مع سوق العراق للاوراق المالية، وهذا تتفق مع الادبيات الاقتصادية، وعدم معنوية الثأثير السلبي لندفقات الاستثار الاجنبي المباشرة، 


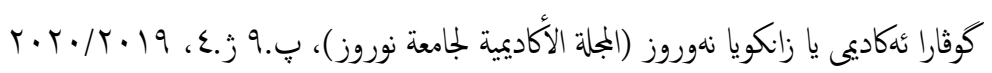

16. Hassan, K. G., \& Sabah, W. (2019). Measuring the impact of some macroeconomic variables on the stock price index in the Iraq Stock Exchange for the period (2006-2015). Academic Journal of Naworoz University, 8(4), 93-106.

17. IIahi, I., Ali, M., \& Jamil, R. A. (2015). Impact of macroeconomic variables on stock market returns: A case of Karachi stock exchange, available at: Electronic copy available at: http://ssrn.com/abstract=2583401.

18. Jabarin, M., Nour, A., \& Atout, S. (2019). Impact of Macroeconomic factors on the market index return at Palestinian stock market. 1-28.

19. Kandir, Y. S. (2008). Macroeconomic variables, firm characteristics and stock returns: Evidence from Turkey. International Research Journal of Finance and Economics, 16, 3545.

20. Khan, Z., Khan, S., \& Rukh, L. (2012). Impact of interest rate, exchange rate and Inflation. International Journal of Economic, 3(5), 142-155.

21. Lee, Y. M., \& Wang, K. M. (2012). Capital mobility and current account imbalance: nonlinear threshold vector auto-regression approach. International Interactions, 38(2), 182-217.

22. Ouma, W. N. (2014). The impact of macroeconomic variables on stock market returns in Kenya. International Journal of Business and Commerce, 3(11), 1-31.

23. Ross, S. A. (1976). The Arbitrage Theory of Capital Market Asset Pricing. Journal of Economic Theory, 13, 341-360.

24. Sharpe, F. W. (1964). Capital Asset Prices: A Theory of Market Equilibrium under Conditions of Risk. Journal of Finance, 19(3): 425-442.

25. The Global Economy, Business and Economic Database, https://www.theglobal economy.com/

26. Umer, M. (2016). Macroeconomic variables impact on stock market performance in the short and long run: A Pakistan perspective. Research. Journal of Finance and Accounting, 7(11), $10-22$.

27. UNICTAD. World Investment Report Series, 2004-2019. United Nations Conference on Trade and Development

http://unctad.org/en/Pages/Publication/

WorldInvestmentReports(1991-2009).aspx.

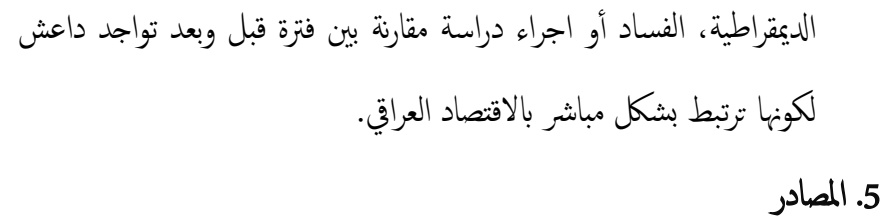

10. Asaad, Z. (2014). The Economic determinants of foreign direct investment inward to Iraq for period (2004-2011). Journal of University of Duhok, 17(1), 192-217.

11. Asaad, Z. A. (2014A). Testing the bank sector at weak form efficiency in Iraq Stock Exchange for period (2004-2014): Empirical research. Journal of Economic Sciences, College of Administration and Economic, Basrah University, 10(37), 57-80.

12. Asaad, Z., Marane, B. M., \& Omer, A. (2015). Testing the efficiency of Iraq Stock Exchange for period (2010-2014): An empirical study. Journal of University of Duhok, University of Duhok, 17(1), 192-217.

13. Asaad, Z. A. (2017). The Influence of the Financial and Real Output Indicators on Malaysian Stock Market. International Cihan Journal of Social Science, 1(1), 4-21.

14. Central Bank of Iraq. (2011). Annual Report 2004-2019, Statistics and Research Department, Iraq. Available at http://www.cbi.iq

15. Faure, A. (2013). Financial System: An introduction. 1st edition, Quoin Institute (Pty) limited. 